\title{
Correlation between HPV sperm infection and male infertility
}

\author{
Yang Yang, Chan-Wei Jia, Yan-Min Ma, Li-Ying Zhou and Shu-Yu Wang
}

Human papillomavirus (HPV) is one of the most common sexually transmitted diseases which comprises a group of small DNA viruses that infect both cutaneous and mucous squamous epithelia. Liquid bead microarray technology (LBMA) were used to evaluate 24 HPV genotypes in confirmed fertile and infertile males of North China so that the effects of HPV infection on semen parameters and relationship with male infertility could be discussed. A total of 1138 subjects were recruited in this study; 142 were HPV-positive (12.48\%). Among 523 confirmed fertile males, only 35 were HPV-positive (6.70\%), and two of them had multiple infections. Among 615 infertile males, 107 were HPV-positive (17.4\%), and 29 of them had multiple infections. Infertile males had a relatively high HPV infection rate compared with confirmed fertile males. Sperm progressive motility (PR) and the normal morphology rate were significantly decreased in HPV-positive subjects. HPV-45, HPV-52, HPV-18, HPV-59 and HPV-16 infections were more frequently in infertile males. Hence, HPV infection is closely related to male infertility which will decrease sperm PR and morphology. HPV-45, HPV-52, HPV-18, HPV-59 and HPV-16 infection seems to be major risk factors.

Asian Journal of Andrology (2013) 15, 529-532; doi:10.1038/aja.2013.36; published online 22 April 2013

Keywords: HPV genotype; human papillomavirus; male infertility

\section{INTRODUCTION}

Human papillomavirus (HPV) is one of the most common sexually transmitted viruses, and males and females from different regions are generally susceptible. ${ }^{1,2}$ More than $120 \mathrm{HPV}$ genotypes are known to distribute in the skin and mucous membranes, including the pubic area, oral cavity and perianal region, ${ }^{3}$ and can be transmitted through skin contact or any other surface contact related to the genital area. HPV infection is commonly associated with genital and non-genital warts or cervical cancer. ${ }^{4}$ Moreover, different HPV genotypes are divided into high-risk types and low-risk types according to the correlation between HPV genotypes and cervical cancer. Previous studies have suggested that more than $50 \%$ of sexually active males or females will have HPV infection in their lives, and the risk of infection could be increased because of multiple sexual partners, sex without condoms and smoking. ${ }^{5-7}$

Male HPV infection has rarely been of concern. HPV infection and its genotypic distribution among Asian males, especially confirmed fertile males, have been seldom reported. ${ }^{8}$ Although HPV infection is highly linked to males and its characteristics of infection types and histological distributions are similar to those in females, most HPV infections among males are benign (e.g., genital warts) with a rare tendency for malignancy. HPV DNA may lie not only in the perianal region and external genitalia, including the penis foreskin, scrotum and glans penis, but also in the urethra, ductus deferens, epididymis or testis. ${ }^{9}$ The infection rate increases greatly when a patient has genital warts, an HPV-positive spouse, or homosexual behaviors. Crossinfection between members of a couple makes HPV chronic and difficult to treat. HPV may infect the semen, ${ }^{10}$ allowing it to affect male fertility in a variety of ways. One study showed that infertile couples with HPV infection have a higher rate of pregnancy loss than do couples without HPV infection during implementation of assisted reproduction technology. ${ }^{11}$ This research demonstrates that HPV infection has significant implications in terms of estimating male fertility or utilizing assisted reproduction technology.

This study aimed to investigate the status of HPV infection in males in North China, understand the population distribution of HPV infection in infertile and confirmed fertile males, and analyze the effects of HPV infection on semen parameters and characteristics of HPV genotype distribution to promote further discussion about the effects of HPV infection on male infertility.

\section{MATERIALS AND METHODS}

\section{Subjects}

Written informed consent was obtained from all subjects, and the study protocol was approved by the ethics committee of our institute. A consecutive case series from Beijing Obstetrics and Gynecology Hospital attached to Capital Medical University was used to obtain the study group. A total of 615 infertile male patients comprised the case group, and 523 confirmed fertile males comprised the control group. The inclusion criterion of the case group was at least 1 year of unprotected sexual intercourse without conception. The exclusion criteria of the case group were as follows: presence of antisperm antibodies, azoospermia, undescended testis, chromosome abnormalities, mumps orchitis or hypergonadotropic/hypogonadotropic hypogonadism. In addition, subjects with a spouse who had tubal, uterine, or cervical abnormalities or bilateral fallopian tube obstruction were excluded. The inclusion criterion of the control group was a spouse who developed a spontaneous pregnancy within 6-8 weeks (i.e., expectant fathers with a baby whose gestational age was $<8$ weeks) and the exclusion criterion was presence of antisperm antibodies. The first step involved inquiry of the subjects' personal history, collection of semen samples for standard semen analysis and detection of HPV infection and genotypes. 


\section{Semen analysis}

All semen samples of subjects were collected by masturbation after 5-7 days of abstinence. Semen parameters (semen volume, $\mathrm{pH}$, sperm concentration, progressive motility (PR) and rate of normal sperm morphology) were analyzed in a thermostat console after semen liquefaction. Procedures were performed with observation of the strict requirements of the WHO Semen Analysis Manual (Version 2010). Saline-washed semen samples were then centrifuged to collect sperm for further analysis.

\section{Detection of HPV infection and genotypes}

The sperm samples were detected and genotyped by liquid bead microarray (LBMA) (Genaco Biomedical Products, Huntsville, AL, USA). LBMA involves a suspension array in which molecular reactions take place on the surface of color-coded beads called microspheres. Microspheres in suspension provide the solid support for binding of the probe. Twenty-four HPV genotypes and target-specific probes were covalently linked to a specific set of microspheres. Polymerase chain reaction (PCR) products labeled by biotin were captured by the probes in a hybridization suspension. A microfluid system delivers the hybridization reaction mixtures in suspension to a dual-laser detection device to detect the hybridization signals associated with each bead and record them.

\section{Statistical analysis}

All data were analyzed with a chi-square test, $t$-test and multiple logistic regression using SPSS statistical software (SPSS for Windows, version 17.0; SPSS, Inc., Chicago, IL, USA).

\section{RESULTS}

\section{Analysis of HPV infection rates}

The average age of the 1138 subjects was 31.16 years (range: $21-48$ years); 142 (12.48\%) were HPV-positive.

Among 615 subjects in the case group, 107 were HPV-positive. The infection rate was $17.40 \%$. Among 523 subjects in the control group, 35 were HPV-positive. The infection rate was $6.70 \%$. The infection rate in the case group was significantly higher than that in the control group $(P<0.001)$.

Among the $107 \mathrm{HPV}$-positive subjects in the case group, 29 (27.1\%) were infected with multiple HPV genotypes. Among the $35 \mathrm{HPV}-$ positive subjects in the control group, two (5.7\%) were infected with multiple HPV genotypes. The difference between the two groups was statistically significant $(P=0.008)$.

\section{Analysis of HPV-positive semen parameters in the case group} In the case group, the sperm PR, vitality and normal sperm morphology rate of the HPV-positive subjects showed statistically significant decreases (Table 1). On the contrary, the semen volume, $\mathrm{pH}$ and sperm concentration showed no significant differences.

\section{Analysis of HPV-positive semen parameters in the control group} In the control group, the sperm PR and normal sperm morphology rate of HPV-positive subjects showed statistically significant decreases (Table 1). On the contrary, the semen volume, $\mathrm{pH}$ and sperm concentration showed no significant differences.

\section{Results of HPV genotyping}

Twenty of the 24 HPV genotypes were found among the 1138 subjects. The most common genotypes were, in decreasing order, HPV-16/45, $-52,-59 / 18,-33,-68$ and $-39 / 42 / 81$ (Table 2).

\section{Different HPV genotype distributions in case and control groups}

The rate of different HPV genotype infections varied in the case and control groups (Figure 1). The most common genotypes in the case group were, in decreasing order, HPV-45, $-16,-52,-18 / 59$ and -33 . The most common genotypes in the control group were, in decreasing order, HPV-68/81, -33 and -39 .

Among the $20 \mathrm{HPV}$ genotypes detected, genotypes HPV-45, -52, $-18,-59$ and -16 in the case group were significantly higher than those in the control group. No significant changes were found in the other 15 genotypes. Risk assessment by multiple regression analysis showed that the infertile odds ratio of HPV-45, HPV-52, HPV-18/59 and HPV-16 infection were 13.9, 12.2, 11.3 and 6.5 respectively (Table 3).

\section{DISCUSSION}

Numerous epidemiologic studies of genital HPV infection in women have been conducted, but data on HPV prevalence and type distribution among men, especially Chinese men, are lacking. In this study, 1138 subjects were selected, and $12.48 \%$ of them had HPV infection; this represents the overall situation of male HPV infection in North China. Meanwhile, by configurating the control group with confirmed fertile males, we found that infertile males had apparently increased HPV or multiple-HPV infection rates, which indicates that HPV infection is an adverse factor affecting male fertility.

Our research data suggest that HPV infection can lower the semen quality, particularly sperm PR and sperm normal morphology; therefore, HPV infection can decrease male fertility or even cause infertility. Some studies speculate that HPV infection lowers sperm vitality; Foresta et al. ${ }^{12,13}$ stated that HPV infection may have the same mechanism as other viruses that infect sperm. They localize at the equatorial region of the sperm head or tail to decrease function and ability of the acrosome, thus affecting the fusion of gametes. However, regardless of whether infertile subjects were HPV-positive in our study, all semen

Table 1 Correlation between HPV positivity and semen parameters. All data are expressed as mean \pm s.d.

\begin{tabular}{|c|c|c|c|c|}
\hline & \multicolumn{2}{|c|}{ Case group } & \multicolumn{2}{|c|}{ Control group } \\
\hline & $H P V(+)(n=107)$ & $H P V(-)(\mathrm{n}=508)$ & $H P V(+)(n=35)$ & $H P V(-)(n=488)$ \\
\hline Volume (ml) & $2.67 \pm 0.79$ & $2.65 \pm 0.63$ & $2.31 \pm 0.72$ & $2.72 \pm 2.59$ \\
\hline $\mathrm{pH}$ & $7.30 \pm 0.36$ & $7.26 \pm 0.31$ & $7.03 \pm 0.32$ & $7.30 \pm 0.35$ \\
\hline Concentration $\left(\times 10^{6} \mathrm{ml}^{-1}\right)$ & $111.31 \pm 78.51$ & $120.96 \pm 85.26$ & $114.42 \pm 61.65$ & $117.52 \pm 84.31$ \\
\hline $\operatorname{PR}(a+b, \%)$ & $20.55 \pm 10.44 *$ & $29.11 \pm 13.66$ & $32.25 \pm 10.00^{\#}$ & $39.22 \pm 12.15$ \\
\hline Morphology (\%) & $4.66 \pm 3.08 *$ & $8.15 \pm 5.05$ & $8.51 \pm 4.21^{\#}$ & $13.01 \pm 4.50$ \\
\hline Virility (\%) & $37.17 \pm 12.53 * *$ & $40.21 \pm 13.93$ & $45.83 \pm 9.84$ & $49.86 \pm 12.49$ \\
\hline
\end{tabular}

Abbreviations: HPV, human papillomavirus; PR, progressive motility.

$* P<0.001$ and $* * P<0.05$ vs. case group HPV $(-)$.

${ }^{\#} P<0.01$ vs. control group HPV (-). 
Table 2 HPV genotyping by HPV-LBMA between the case and control groups

\begin{tabular}{lccr} 
& \multicolumn{3}{c}{ Samples positive for HPV, $\mathrm{n}(\%)$} \\
\cline { 2 - 4 } HPV genotype & Control group & Case group & \multicolumn{1}{c}{ Total } \\
\hline HPV (any) & $38(22.22)$ & $133(77.78)$ & $171(100)$ \\
HPV-6 & $2(1.17)$ & $5(2.92)$ & $7(4.09)$ \\
HPV-11 & $2(1.17)$ & $3(1.75)$ & $5(2.92)$ \\
HPV-16 & $2(1.17)$ & $15(8.77)$ & $17(9.94)$ \\
HPV-18 & $1(0.58)$ & $13(7.60)$ & $14(8.19)$ \\
HPV-31 & $1(0.58)$ & $6(3.51)$ & $7(4.09)$ \\
HPV-33 & $4(2.34)$ & $9(5.26)$ & $13(7.60)$ \\
HPV-39 & $3(1.75)$ & $7(4.09)$ & $10(5.85)$ \\
HPV-40 & $1(0.58)$ & $2(1.17)$ & $3(1.75)$ \\
HPV-42 & $2(1.17)$ & $8(4.68)$ & $10(5.85)$ \\
HPV-43 & 0 & $1(0.58)$ & $1(0.58)$ \\
HPV-45 & $1(0.58)$ & $16(9.36)$ & $17(9.94)$ \\
HPV-51 & 0 & $1(0.58)$ & $1(0.58)$ \\
HPV-52 & $1(0.58)$ & $14(8.19)$ & $15(8.77)$ \\
HPV-53 & $1(0.58)$ & $5(2.92)$ & $6(3.51)$ \\
HPV-54 & $2(1.17)$ & $2(1.17)$ & $4(2.34)$ \\
HPV-59 & $1(0.58)$ & $13(7.60)$ & $14(8.19)$ \\
HPV-66 & 0 & $4(2.34)$ & $4(2.34)$ \\
HPV-68 & $7(4.09)$ & $5(2.92)$ & $12(7.02)$ \\
HPV-70 & 0 & $1(0.58)$ & $1(0.58)$ \\
HPV-81 & $7(4.09)$ & $3(1.75)$ & $10(5.85)$ \\
\hline
\end{tabular}

Abbreviations: HPV, human papillomavirus; LBMA, liquid bead microarray.

quality parameters were obviously decreased, indicating that there are factors other than HPV infection that lead to infertility.

We found that the most common HPV genotypes among the 1138 subjects, in decreasing order, were HPV-16/-45, -52, -59/-18, -33, -68 and $-39 /-42 /-81$. However, the HPV genotypes of infertile males were different: $-45,-16,-52,-18 /-59$ and -33 . HPV-45, $-52,-18,-59$ and -16 have close relationships with infertility because they are both cervical cancer and male infertility related high-risk type.

Perino ${ }^{11}$ reported that couples who underwent assisted reproductive technology cycles experienced an increased risk of pregnancy loss when HPV DNA testing was positive in the male partner, compared with non-infected patients. In addition,

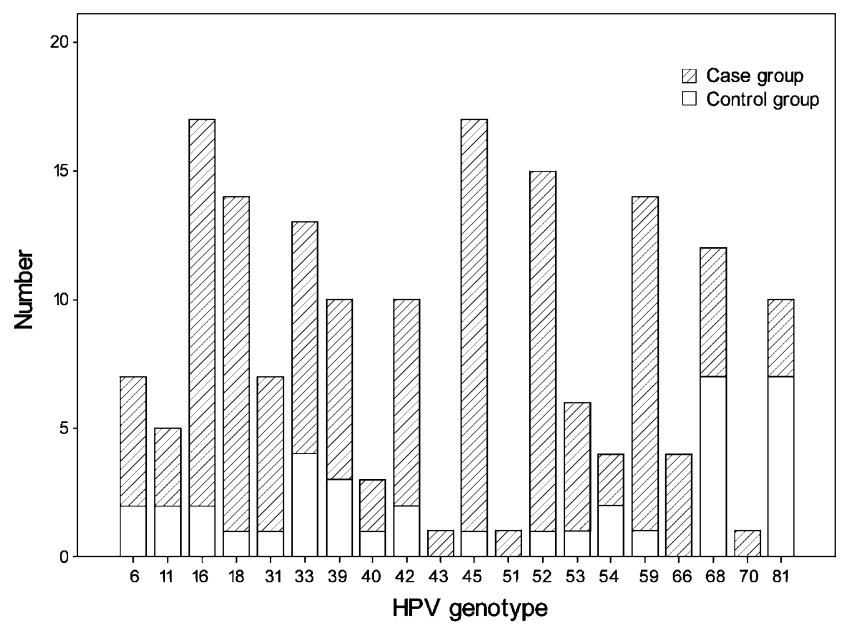

Figure 1 Different HPV genotype distributions in case and control groups. HPV, human papillomavirus.
Table 3 Risk assessment by HPV genotyping with HPV-LBMA

\begin{tabular}{lcrl}
\hline HPV genotype & $P$ & OR & \multicolumn{1}{c}{$95 \% \mathrm{Cl}$} \\
\hline HPV-16 & 0.016 & 6.510 & $1.482-28.611$ \\
HPV-18 & 0.024 & 11.272 & $1.470-86.460$ \\
HPV-45 & 0.010 & 13.943 & $1.843-105.497$ \\
HPV-52 & 0.028 & 12.160 & $1.594-92.784$ \\
HPV-59 & 0.048 & 11.272 & $1.470-86.460$ \\
\hline
\end{tabular}

Abbreviations: $\mathrm{Cl}$, confidence interval; HPV, human papillomavirus; LBMA, liquid bead microarray; OR, odds ratio.

HPV-51 and HPV-52 were most frequently identified in the male partner of infertile couples. In our study, all subjects from the control group were confirmed fertile males, and semen samples were collected after week 6 to 8 of pregnancy. We performed follow-up investigations of $35 \mathrm{HPV}$-positive subjects until their week 16 to 20 of pregnancy and found that four of their spouses showed abortion symptoms (abdominal pain, vaginal bleeding) that were relieved after obstetric therapy, no early abortion or suspension of embryo growth. The most common genotypes of confirmed fertile males were HPV-68, $-81,-33$ and -39 . We conjectured that the effect of these genotypes on pregnancy is limited; in other words, HPV infection has little to do with the early results of natural conception. Further studies with increased numbers of samples or prolonged follow-up times are needed.

In conclusion, HPV infection is a risk factor for male infertility. It decreases sperm PR and the normal morphology rate, leading to decreased male fertility or even infertility. Among the male HPV infection genotypes, HPV-45, -52, -18/59 and -16 have close relationships with infertility and deserve more attention.

\section{AUTHOR CONTRIBUTIONS}

SYW designed the research and wrote the paper. YY participated in the design of the research, carried out the studies, performed statistical analysis and drafted the manuscript. CWJ and YMM participated in the design of the study. LYZ participated in the statistical analysis. All authors read and approved the final manuscript.

\section{COMPETING FINANCIAL INTERESTS}

The authors declare no competing financial interests.

1 Palefsky JM. Human papillomavirus-related disease in men: not just a women's issue $J$ Adolesc Health 2010; 46(4 Suppl): S12-9.

2 Giuliano AR, Lee JH, Fulp W, Villa LL, Lazcano E et al. Incidence and clearance of genital human papillomavirus infection in men (HIM): a cohort study. Lancet 2011; 377: 932-40

3 Munoz N, Bosch FX, de Sanjose S, Herrero R, Castellsague X et al. International Agency for Research on Cancer Multicenter Cervical Cancer Study Group. Epidemiologic classification of human papillomavirus types associated with cervical cancer. N Engl J Med 2003; 348: 518-27.

4 Robinson D, Coupland V, Møller H. An analysis of temporal and generational trends in the incidence of anal and other HPV-related cancers in Southeast England. Br J Cancer 2009; 100: 527-31.

5 Vardas E, Giuliano AR, Goldstone S, Palefsky JM, Moreira ED Jr et al. External genital human papillomavirus prevalence and associated factors among heterosexual men on 5 continents. J Infect Dis 2011; 203. 58-65.

6 Nyitray AG, Carvalho da Silva RJ, Baggio ML, Lu B, Smith D et al. Age-specific prevalence of and risk factors for anal human papillomavirus (HPV) among men who have sex with women and men who have sex with men: the HPV in men (HIM) study. $J$ Infect Dis 2011; 203: 49-57. 
7 Goldstone S, Palefsky JM, Giuliano AR, Moreira ED Jr, Aranda C et al. Prevalence of and risk factors for human papillomavirus (HPV) infection among HIV-seronegative men who have sex with men. J Infect Dis 2011; 203: 66-74.

8 Barzon L, Militello V, Pagni S, Franchin E, Dal Bello F etal. Distribution of human papillomavirus types in the anogenital tract of females and males. J Med Virol 2010; 82: 1424-30.

9 Rintala MA, Pollanen PP, Nikkanen VP, Grenman SE, Syrjanen SM. Human papillomavirus DNA is found in the vas deferens. J Infect Dis 2002; 185: 1664-7.

10 Rintala MA, Grenman SE, Pollanen PP. Detection of high-risk HPV DNA in semen and its association with the quality of semen. Int J STD AIDS 2004; 15: 740-3.
11 Perino A, Giovannelli L, Schillaci R, Ruvolo G, Fiorentino FP et al. Human papillomavirus infection in couples undergoing in vitro fertilization procedures: impact on reproductive outcomes. Fertil Steril 2011; 95: 1845-8.

12 Foresta C, Patassini C, Bertoldo A, Meneqazzo M, Francavilla F et al. Mechanism of human papillomavirus binding to human spermatozoa and fertilizing ability of infected spermatozoa. PLoS ONE 2011; 6: e15036.

13 Foresta C, Pizzol D, Moretti A, Barzon L, Palu G et al. Clinical and prognostic significance of human papillomavirus DNA in the sperm or exfoliated cells of infertile patients and subjects with risk factors. Fertil Steril 2010; 94: 1723-7. 